\title{
Importancia de la edad como factor de riesgo en la orientación del programa de prevención del cáncer de cuello uterino
}

\author{
Antonio González Mazuelo*; Néstor Oswaldo Villota Cadena*
}

RESUMEN: Se hizo un análisis del comportamiento de algunas variables del programa de prevención y detección de cáncer Cérvicouterino en la Empresa Social del Estado (E.S.E.)- Metrosalud durante los años 1991 a 1994 en relación con la edad.

Se consolidaron los cuadros de salida que rutinariamente se elaboran con respecto al número de citologías tomadas, número de citologías anormales, resultados de la biopsia dirigida por Colposcopia y número y estadío de los cánceres diagnosticados. Cada una de estas variables se analizó con base en la edad para determinar la importancia de la misma.

Se observó una alta proporción de mujeres jóvenes menores de 25 años que acudieron a la toma de citología, con respecto al grupo de 35 a 44 años, que es en el que se encontró una mayor frecuencia relativa de NIC III y un incremento notorio en la frecuencia de cáncer.

Se concluye la importancia de lograr un incremento en la cobertura citológica en dicho grupo de edad a fin de lograr un resultado favorable en la ejecución del programa de prevención al mediano plazo.

SUMMARY: We had an analysis of behavior of some variables of program "Cervical Cancer: detection and prevention" at the Enterprise Social of the State (Empresa Social del Estado E.S.E.)-Metrosalud between 1991 and 1994 in relation with the age.

We consolidated in the oultet's pictures that routinely we elaborated regarding: number of citologies, number of abnormal citologies, result of the colposcopically directed biopsies and number and stage of invasive cervical carcinoma. Each one of these variables we analyzed in basis of the age, in order to determine the importance of itself.

We observed a high proportion of citologies taked in younger women (minor of 25 years old) regarding to the group, with major frequency of CIN III and with an increase well-known in the frequency of cancer (35-44 years old).

We concluded the importance to increase the number of pap smears in the group of 35-44 years old in order to achieve an favorable result in the performance the program of prevention in short time.

\section{Introducción}

Hace 19 años ya que el cáncer en general se constituyó en la primera causa de muerte entre la población femenina del país (1).

Datos recientes publicados por el Instituto Nacional de Cancerología (I.N.C.) muestran los cánceres de cuello uterino y de mama como la segunda y tercera causas de muerte por cáncer en la mujer colombiana, con tasas de mortalidad estandarizadas por 100.000 de 6.49 y 5.77 respectivamente, sólo superadas por el carcinoma de estómago, primera causa de muerte por cáncer en la población general (2). Así, el cáncer de origen ginecológico sigue teniendo un peso importante en el estado de salud de la mujer colombiana.

La técnica del frotis de Papanicolau, un procedimiento sencillo y barato con casi 70 años de presencia en el

Profesores de Gineco-Obstetricia. Universidad Pontificia Bolivariana, Medellín. conocimiento médico y más de 40 años de aceptación general, ha demostrado que, practicado con regularidad, contribuye eficazmente en la prevención secundaria del carcinoma de cuello uterino. Programas de tamizaje citológico bien conducidos han demostrado en países desarrollados, un notorio impacto sobre la mortalidad por esta enfermedad, disminuyendo las tasas de incidencia de la misma al eliminar eficazmente las formas precursoras (N.I.C.) (3).

30 años han transcurrido desde que la técnica de citología cérvico-uterina fue introducida en el país, sin embargo las estadísticas muestran que el uso de la prueba es deficiente. La encuesta nacional de salud realizada en Colombia en 1991 mostró en su capítulo sobre aspectos varios de la salud femenina que el $65.9 \%$ de la población femenina tiene conocimiento de la citología vaginal (53.1\% a nivel rural) pero sólo la habían practicado alguna vez en su vida $46.2 \%$ de ellas ( $39.4 \%$ a nivel rural) y $51.2 \%$ de las mismas con cierta regularidad; quiere decir esto que en Colombia sólo 1/6 parte de la población femenina conoce y practica con la regularidad adecuada la citología cérvico uterina (4). 
Son los grandes centros urbanos donde la mujer tiene mejor conocimiento de la prueba y donde más se practica con cierta frecuencia alcanzándose alguna cobertura importante. Pero es también en ellas donde se están presentando el mayor número de casos y donde la enfermedad alcanza las más altas tasas de mortalidad (5).

Colombia es consciente desde 1990 que el tamizaje casual o el que llevan a cabo permanentemente grupos seleccionados de pacientes, no lograría impactar de forma significativa la incidencia y la mortalidad por cáncer cérvico-uterino. Desde ese año se implementó a nivel nacional un programa estructurado, sistemático y relativamente bien financiado de tamizaje citológico; sus efectos no podrán ser evaluados antes de transcurridos al menos 10 años.

El programa para ser efectivo debe universalizar el uso de la citología, en otras palabras, incrementar en forma notoria la cobertura y optimizar el seguimiento. El análisis del desarrollo del programa en Medellín ha mostrado que este ha sido lento por graves limitaciones de recursos técnicos y humanos, porque las instituciones no tienen suficiente personal de enfermería o de citotécnicos para atender un volumen importante, a gran escala, de usuarias. En cuatro años de ejecución sólo se alcanzó el $8.6 \%$ de las citologías propuestas en la meta nacional para ese mismo período (*).

Surge entonces la preocupación de como optimizar aún más los logros del programa a mediano plazo, evitando las limitaciones detectadas.

La alternativa es orientar los recursos y el esfuerzo hacia aquella población de mujeres con mayor riesgo de enfermar de cáncer de cuello uterino.

Siguiendo las recomendaciones de A.B. Miller: "el conocimiento sobre los factores de riesgo no proporcionan una estrategia práctica para la prevención de la enfermedad... el factor de riesgo más importante para determinar el grupo prioritario para la detección es la edad. Existe además una relación inversa entre la situación socioeconómica y el riesgo de cáncer de cuello uterino en todos los países" (6); más adelante aconseja... "los grupos destinatarios de la detección deben ser las mujeres con riesgo definido, en particular la edad y el grupo socioeconómico bajo. La detección debe dirigirse a los grupos de edad en los que el cáncer cérvico-uterino es corriente empezando unos años antes de que la incidencia de cáncer alcance niveles apreciables" (6).

La Empresa Social del Estado (E.S.E.)-Metrosalud es una institución al servicio de la población de más bajos recursos por tanto el análisis debe orientarse hacia que grupo de edad debe priorizarse la atención.

Con el fin de proporcionar una recomendación a este respecto se hizo un análisis retrospectivo de los datos recolectados hasta ahora en los cuatro años de ejecución del programa respecto a una sola variable: la edad. Quisimos observar como se comportaban la toma de citología, la frecuencia de NIC o cáncer en relación con la edad; se quiso también en base a los datos, definir las

(*) Informe interno aún no publicado. medias de la edad a la que se presentan las diferentes formas de la enfermedad en el Area Metropolitana del Valle de Aburrá.

El resultado de dicho análisis se presenta en el siguiente informe.

\section{Materiales y métodos}

Para la realización del presente análisis se examinaron los archivos informáticos que guarda la (E.S.E.)Metrosalud de la ejecución del programa de detección y control del cáncer de cuello uterino desde junio de 1991 hasta diciembre de 1994. Los datos están consignados en cuadros fijos proporcionados por el I.N.C. y corresponden a las formas... $1.1,1.2$ y 1.3 .

Dichos cuadros contienen información semestral desde junio de 1991 basados en grupos de edad pre-establecidos por las normas técnicas del programa sobre: citologías tomadas, citologías anormales, pacientes estudiados por Colposcopia y resultados de la biopsia dirigida.

Desde Enero de 1994 la información se almacenó de forma computarizada en una base de datos individual creada en el programa epidemiológico de la O.M.S. epiinfo Versión 5. Los datos de población utilizados en el análisis fueron tomados del departamento de informática de la E.S.E. Metrosalud basados en el año 1993 con proyecciones del censo del año 85. La organización de los datos proporciona cuatro cuadros de salida (tablas 2, 3 y 4 ).

Se consideraron en el análisis las siguientes variables:

1. Distribución porcentual de la población femenina mayor de 15 años por grupo de edad.

2. Proporción de citologías tomadas en cada grupo de edad (F1).

$$
\mathrm{F} 1=\frac{\text { citologías }(\mathrm{B})}{\text { población }(\mathrm{A})} \times 100 \text {, en cada grupo de edad }
$$

3. Proporción de citologías anormales en cada grupo de edad.

$\mathrm{F} 2$ = citologías anormales (C), en cada grupo de edad citologías (B)

4. Proporción de casos de cada forma de la enfermedad por citologías tomadas y por grupo de edad.

$$
\text { F3 }=\frac{\text { casos de NIC o Cáncer (D) }}{\text { citologías (B) }} \times \begin{gathered}
\text { 1000, en cada grupo } \\
\text { de edad }
\end{gathered}
$$

El cálculo en (F1) representa un valor aproximado de la "cobertura" de citología en cada grupo de edad al relacionar el número de citologías tomadas en cada grupo de edad según la población total de dicho grupo.

El cálculo en (F2) representa a la frecuencia de anormalidad en cada grupo de edad.

El cálculo en (F3) representa una aproximación a la "incidencia" de cada estado de la enfermedad, al relacionar el número de casos de cada estado de la enfermedad según el volumen de citologías realizadas en cada grupo de edad. 
Tabla 2

CITOLOGIAS TOMADAS Y CITOLOGIAS ANORMALES POR GRUPO DE EDAD

\begin{tabular}{|c|c|c|c|c|}
\hline Edad & \multirow{2}{*}{$\begin{array}{c}\text { Total citologías } \\
\text { tomadas (B) }\end{array}$} & \multicolumn{2}{|c|}{$\begin{array}{c}\text { Citologías } \\
\text { anormales }\end{array}$} & \multirow{2}{*}{$\begin{array}{c}\text { Total citologías } \\
\text { anormales (C) }\end{array}$} \\
\cline { 3 - 4 } & & VPH & NIC & \\
\hline $15-24$ & 13.641 & 101 & 127 & $228 \%$ \\
$25-34$ & 19.588 & 124 & 240 & $364 \%$ \\
$35-44$ & 10.513 & 71 & 149 & $220 \%$ \\
$450+$ & 11.473 & 38 & 153 & $191 \%$ \\
\hline Total & 55.215 & 334 & 669 & $1.003 \%$ \\
\hline
\end{tabular}

Tabla 3

RESULTADO DE LA BIOPSIA DIRIGIDA POR COLPOSCOPIA EN PACIENTES CON CITOLOGIA ANORMAL POR GRUPO DE EDAD

\begin{tabular}{|c|c|c|c|c|c|c|c|}
\hline Edad & $\begin{array}{c}\text { Negativo } \\
\text { Cervicitis }\end{array}$ & $\begin{array}{c}\text { VPH } \\
\text { (D1) }\end{array}$ & $\begin{array}{c}\text { NIC I } \\
\text { (D2) }\end{array}$ & $\begin{array}{c}\text { NIC II } \\
\text { (D3) }\end{array}$ & $\begin{array}{c}\text { NIC III } \\
\text { (D4) }\end{array}$ & $\begin{array}{c}\text { Cáncer } \\
\text { (D5) }\end{array}$ & Total \\
\hline $15-24$ & 13 & 113 & 48 & 37 & 16 & 1 & 228 \\
$25-34$ & 43 & 126 & 56 & 46 & 84 & 9 & 364 \\
$35-44$ & 32 & 66 & 33 & 17 & 51 & 21 & 220 \\
45 ó & 36 & 51 & 25 & 15 & 30 & 34 & 191 \\
\hline Total & 124 & 356 & 162 & 115 & 181 & 65 & 1.003 \\
\hline
\end{tabular}

Tabla 4

ESTADIOS DE LOS CANCERES CERVICO-UTERINOS DIAGNOSTICADOS POR GRUPO DE EDAD

\begin{tabular}{|l|c|c|c|c|c|}
\hline \multirow{2}{*}{ Edad } & Temprano & \multicolumn{3}{|c|}{ Avanzado } & \multirow{2}{*}{ Total } \\
\cline { 2 - 5 } & I & II & III & IV & \\
\hline $15-24$ & 1 & 0 & 0 & 0 & 1 \\
$25-34$ & 9 & 0 & 0 & 0 & 9 \\
$35-44$ & 16 & 2 & 3 & 0 & 21 \\
45 Ó + & 9 & 8 & 17 & 0 & 34 \\
\hline Total & 35 & 10 & 20 & 0 & 65 \\
\hline
\end{tabular}

Se aplicaron las fórmulas matemáticas mencionadas a los cuadros de salida y sus resultados se graficaron.

5. Distribución por estadio de los cánceres diagnosticados en cada grupo de edad.

6. Media y Moda de la edad de cada forma de la enfermedad.

La media y su desviación estándar la calculó el programa informático usado en el computador; se obtuvo esta variable según el estado de la enfermedad, para la población sometida a tamizaje y para la población con citología anormal sometida a colposcopia.
Para la moda se pidió al programa de computador un listado del número de casos para cada edad y estado de la enfermedad, de la población sometida a tamizaje y de la población con citología anormal sometida a colposcopia, y los datos fueron graficados en forma de distribución de frecuencias.

\section{Resultados}

La oficina de estadística de la ESE-Metrosalud Medellín calculó para 1993 la población de la ciudad en 1.777.128 habitantes: el 52.96\% de la misma corresponde al sexo femenino; 689.332 mujeres eran mayores de 15 años; $63.06 \%$ están asignadas para la atención a Metrosalud y corresponde a sectores de nivel socioeconómico bajo sin vínculo laboral estable y equivale a 434.695 mujeres.

Dicha población se distribuyó por edad según grupos preestablecidos en el programa. Se agrupó la población con 45 años o más porque epidemiológicamente se comporta de manera semejante en relación con la enfermedad según un análisis estadístico previo y reportes de investigaciones regionales sobre incidencia y mortalidad por carcinoma invasor.

Muestra la distribución por edad de la población femenina mayor de 15 años asignada a Metrosalud y que es la población objetivo del programa nacional de tamizaje. Se observó que la población menos numerosa es el grupo 35 a 44 años en el período de transición entre la época reproductiva y el climaterio.

El gráfico No. 2 muestra la distribución por edad de las citologías tomadas donde se resalta como un $60.18 \%$ de las mismas se toman en la población joven menor de 35 años y una cuarta parte $(24.7 \%)$ en menores de 25 años.

Muestra la proporción de citologías tomadas en cada grupo de edad. No puede hablarse de cobertura en el sentido estricto de la palabra puesto que no se discriminan en este análisis de las 55.215 citologías tomadas en el período cuantas eran de primera vez, se quería conocer que grupo de edad se hizo más citologías. Como se observa es el grupo de edad entre 25 y 34 años quien presenta la frecuencia más alta y hay una notoria caída en los grupos de edad posteriores.

\section{Gráfico 2}

\section{DISTRIBUCION DE LA TOMA CITOLOGICA} por grupo de edad

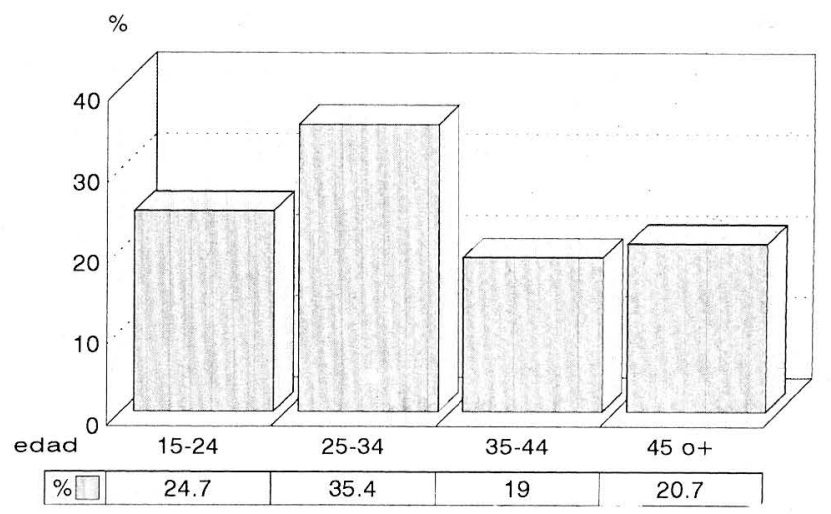


Gráfico 4

PROPORCION DE CITOLOGIAS ANORMALES

por grupo de edad

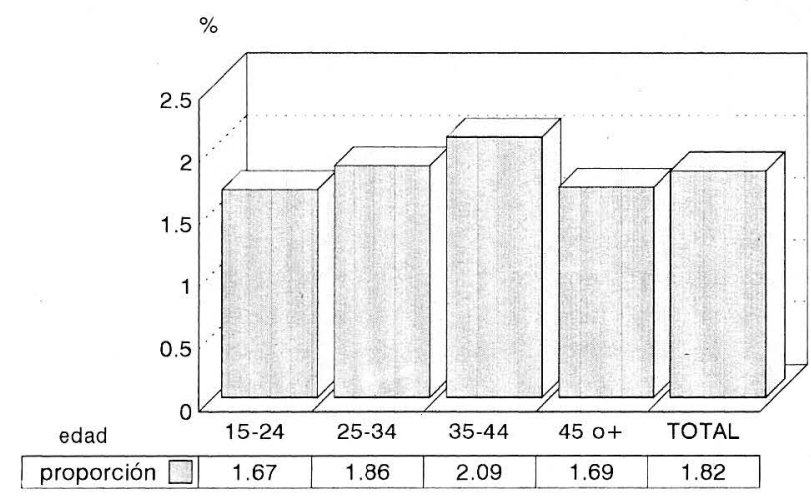

El gráfico 4 muestra la frecuencia de citologías anormales por grupo de edad. El promedio de anormalidad citológica es de $1.82 \%$, bajo para los estándares que estipulan 2 a $3 \%$. Como se observa en el grupo entre 35 y 44 años al que presenta la frecuencia más alta de anormalidad citológica mientras que los demás grupos son relativamente semejantes.

El gráfico 5 muestra la frecuencia de cada tipo de lesión histológica relativo al número de citologías que cada grupo de edad se toma. Esta proporción da una medida indirecta de la incidencia de cada estado de la enfermedad dependiente de la edad. Como se observa las lesiones por papilomavirus, NIC I, NIC II son constantes en cada grupo de edad excepto en el grupo de edad mayor o igual a 45 años donde hubo un descenso estadísticamente significativo; por el contrario las lesiones NIC III se incrementan progresivamente hasta alcanzar su mayor frecuencia entre los 35-44 años para luego disminuir. La frecuencia de cáncer de cuello uterino crece progresivamente con la edad, con un incremento de 4.45 veces en el grupo de 35 a 44 años y 6.58 veces en el grupo de 45 años o más.

\section{Gráfico 5}

\section{TASA DE FRECUENCIA DE CADA TIPO DE LESION} HISTOLOGICA

por 1000 citologías y por grupo de edad

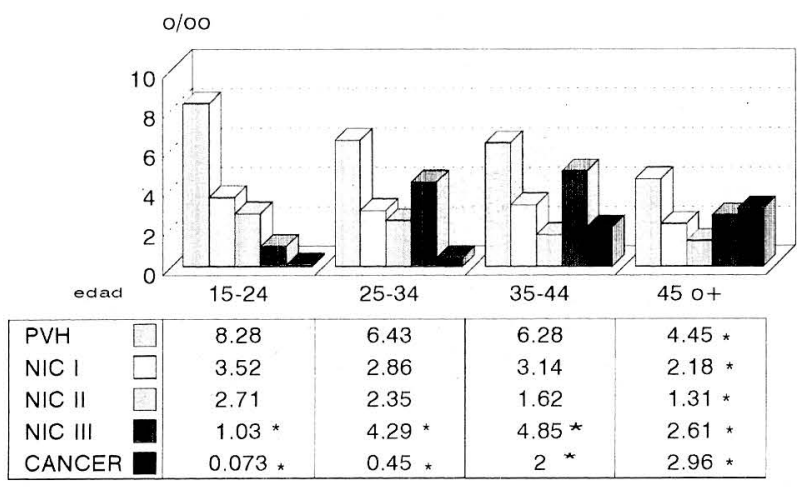

(*) Valores estadísticamente significativos
La prueba de t de Student de significancia estadística confirmaron que las frecuencias de V.P.H., NIC I y NIC II no muestran mayor variación con la edad entre los 15 y 44 años.

Confirma un incremento significativo de los carcinomas invasores a partir de los 25 años. Entre los 15 y 34 años fueron en un 100\% diagnosticados en estadio I donde la sobrevida a 5 años es mayor del $90 \%$.

El grupo de 35 a 44 años muestra la presencia de formas avanzadas de cáncer de cuello uterino y después de los 45 años la mayoría son diagnosticados en estadios avanzados donde la sobrevida se reduce notoriamente.

La Tabla 5 presenta la media y la desviación estándar de la edad para cada tipo de lesión histológica, ellas permiten inferir las distancias en años entre las lesiones por papilomavirus, NIC I y NIC II con el NIC III y entre este último y el cáncer. Se observa la similitud en el promedio de edad de todas las lesiones preinvasoras que muestran valores muy cercanos entre los 30 y los 35 años: sólo el cáncer se alejó significativamente con un intervalo de 13 años entre la media de edad de este (48 años) y la del NIC III (35 años).

Inspirados en otras publicaciones se consideró que la moda pudiera ser un mejor estadígrafo para evaluar la tendencia central de la edad para las lesiones preinvasoras. Muestra la distribución de frecuencias por edad y la moda de una muestra representativa de la población de 55.215 citologías tomadas; la moda fue de 30 años para la población sometida a tamizaje. Muestra la distribución de frecuencias por edad y la moda de la población con citología anormal que fueron investigadas por colposcopia (1003); la moda fue de 29 años.

Muestran la distribución de frecuencias por edad y la moda para cada lesión histológica: P.V.H., NIC I, NIC II y NIC III.

La distribución de frecuencias por edad para papilomavirus y NIC I se sitúan hacia grupos de edad más jóvenes con modas a $\operatorname{los} 23$ y 25 años; en cambio la distribución de frecuencias por edad para NIC II y NIC III se sitúan hacia edades intermedias con modas entre los 28 y 31 años.

Tabla 5

MEDIA Y DESVIACION ESTANDAR DE LA EDAD PARA CADA TIPO DE LESION HISTOLOGICA Y EL INTERVALO EN AÑOS ENTRE CADA UNA DE ELLAS

\begin{tabular}{|l|c|c|}
\hline $\begin{array}{l}\text { Tipo de } \\
\text { lesión }\end{array}$ & $\begin{array}{c}\text { Media y } \\
\text { Desviación estándar }\end{array}$ & $\begin{array}{c}\text { Intervalo } \\
\text { en años }\end{array}$ \\
\hline PVH & $32.12 \pm 11.91$ & \multirow{2}{*}{3} \\
\cline { 1 - 2 } NIC I & $32.30 \pm 12.28$ & \multirow{2}{*}{13 años } \\
\hline NIC II & $30.61 \pm 10.51$ & \multirow{2}{*}{13 años } \\
\hline NIC III & $35.78 \pm 11.00$ & \\
\hline Cáncer & $48.46 \pm 14.16$ & \\
\hline
\end{tabular}


De esta manera se aprecian diferentes tendencias centrales en la edad de las distintas lesiones preinvasoras. De su comparación con las tendencias de la población con citología anormal se observa la predominancia de la infección por PVH y NIC I en mujeres jóvenes.

\section{Discusión}

Siendo prevenible y un importante problema de salud pública en Colombia era fundamental tomar la decisión de adelantar un programa nacional de prevención, pero sus posibilidades estuvieron y están limitadas por la disponibilidad de instalaciones y recurso humano; no hay con que ofrecer una cobertura suficiente a toda la población expuesta. Las metas propuestas en cobertura y seguimiento están lejos de alcanzarse.

"Desde el punto de vista técnico el empleo de la citología vaginal para detectar las lesiones precursoras del cáncer de cuello uterino es muy eficaz y puede evitar la morbilidad y la mortalidad provocadas por esta enfermedad pero para que sea eficaz en el plano de la población general es preciso organizar cuidadosamente programas de detección" (7).

Siendo así puede ser una decisión importante priorizar la atención y volcar todos los recursos disponibles hacia aquel grupo de mujeres que puede generar resultados importantes en el mediano plazo. Se recomienda que los dos parámetros para guiar esta decisión sea considerar el nivel socioeconómico y la edad como los principales factores de riesgo a nivel de la población general.

Ante la imposibilidad de lograr la cobertura propuesta en toda la población expuesta habrá que priorizar el grupo más sensible y donde el programa arroje los mejores resultados y sea más eficaz.

"A nivel de Salud Pública parece que el factor de riesgo más importante para determinar el grupo prioritario para la detección es la edad" (8).

Por lo tanto se impone el estudio de este primerísimo factor de riesgo y de su análisis extraer conclusiones que permitan orientar el programa.

El análisis de los datos del presente informe muestran al grupo de mujeres entre los 35 y 44 años como el prioritario para la atención dentro del programa de prevención. En dicho grupo de edad se detectó la frecuencia más alta de anormalidades en el resultado de la citología y a su vez la frecuencia más alta de lesiones NIC III. De la misma manera en dicho grupo de edad se detectó un incremento significativo en la frecuencia de cáncer y a su vez es el grupo en que empiezan a hacer su aparición formas avanzadas de la enfermedad. Parece deducirse que las lesiones preinvasoras (específicamente NIC III) a esta edad son las potencialmente más dispuestas al cáncer y la que deben diagnosticarse y tratarse con mayor diligencia y así prevenir el cáncer avanzado en edades posteriores.

Los estudios de incidencia de cáncer cérvico-uterino en Antioquia muestran tasas muy bajas de incidencia por debajo de los 30 años de edad (5.8 x 100.000 mujeres) con un aumento progresivo y significativo después de los 30 años (64.3 x 100.000 mujeres).

Del mismo modo los estudios de mortalidad realizados en el país y en Antioquia muestran una mortalidad muy baja por debajo de los 30 años $(0.85 \times 100.000$ mujeres) y un incremento significativo después de los mismos (15.9 a $45 \times 100.000 \mathrm{~m})$.

De este modo el programa debe intensificar sus actividades en el grupo de mujeres mayores de 25 años, una década antes del grupo en que el cáncer presenta un incremento significativo en su incidencia y lograr una cobertura del 100\% en el grupo de 35 a 44 años, meta que se facilita pues es el grupo menos numeroso de toda la población objeto del programa.

El presente informe muestra una alta proporción de citologías tomadas en mujeres en edad reproductiva $(60 \%)$ y particularmente en mujeres menores de 25 años. Debe hacerse un replanteamiento sobre la utilidad que esto pueda tener para los objetivos del programa de prevención. Como se menciona la probabilidad de padecer cáncer de cuello uterino o de morir por esta causa es muy baja antes de los 25 años.

"En la mayoría de los países la incidencia de cáncer cérvico-uterino es muy baja por debajo de los 25 años de edad" (9).

E1 tamizaje citológico en las mujeres jóvenes pareciese tener un efecto educativo a largo plazo pero no es así, se diagnosticó un número elevado de lesiones PVH y NIC I que consumen recursos en diagnóstico y tratamiento, la mayoría de las cuales no progresarán al cáncer y por el contrario no está demostrado que estas mujeres continuarán el tamizaje citológico después de los 35 años cuando el riesgo se incrementa significativamente, pues han asociado la prueba a los eventos reproductivos (maternidad, planificación familiar, etc).

En nuestro medio según la opinión general y de algunos artículos científicos, se quiere priorizar a las mujeres jóvenes en el programa de tamizaje por un no muy bien evaluado aumento de la incidencia de cáncer cérvico-uterino en este grupo de edad, producto de "agresiones virales altamente oncógenas".

Una revisión reciente sobre si ha cambiado la naturaleza del cáncer cérvico-uterino en la mujer joven trae varias conclusiones interesantes: 1) los cánceres cérvicouterinos de alta agresividad no es un fenómeno nuevo, 2) en los últimos 25 años la incidencia y la correspondiente mortalidad por cáncer cervical parece haberse incrementado en mujeres por debajo de los 35 años de edad, sin embargo enfatizan, no es un hallazgo universal y parece limitado a las poblaciones objeto de los estudios, 3) no puede decirse que el pronóstico en la mujer joven sea significativamente diferente de las cohortes de mayor edad y los reportes de lesiones de alta agresividad y rápido crecimiento son más bien anecdóticas y basados en pequeño número de pacientes referidos a la práctica de uno y otro autor (10).

El análisis de este estudio parece confirmar los conocimientos epidemiológicos sobre cáncer de cuello uterino en mujeres menores de 25 años. Sólo reportamos un caso, el de una mujer de 23 años G2P2V2 con historia clínica No. 129339 que consultó por un resultado de citología Pap IV; la colposcopia altamente significativa fue insatisfactoria y el curetaje endocervical positivo. Se hizo, como estaba indicado una conización diagnóstica 
el 17 de marzo de 1993; el informe de patología del mismo (No. 24603) reportó infección por papiloma virus humano y carcinoma microinvasor de origen escamoso con profundidad menor de $3 \mathrm{~mm}$. Un caso en 13.641 citologías tomadas en el grupo de 15 a 24 años o sea 0.07 casos por cada 100 citologías en dicho grupo de edad.

En contradicción con las recomendaciones anotadas de priorizar las mujeres de mayor edad, la frecuencia de citología disminuye progresivamente luego de los 35 años lo cual es un contrasentido desde el punto de vista epidemiológico. Si se reconoce por las estadísticas el riesgo incrementado a medida que la mujer envejece el programa debía propugnar por incrementar o al menos mantener la cobertura después de los 45 años.

Los años de separación en las medias de frecuencia entre NIC III y cáncer (13 años) son semejantes a los reportados en la literatura y permite suponer que no hay un cambio o aceleración en la historia natural de la enfermedad en nuestro medio. (11).

Citando al Dr. Disala: "la edad media del NIC III previo al cáncer (10 a 15 años) justifica los programas de tamizaje con intervalos superiores a un año" (12).

El presente análisis soporta el propósito nacional de hacer un tamizaje de seguimiento con intervalos de 3 años. Esto permite optimizar la utilización de los recursos sin exponer a las usuarias a un riesgo en caso dado de que la enfermedad hubiera acelerado su curso natural.

Llama la atención en este informe el comportamiento semejante de las lesiones por papilomavirus, NIC I y NIC II y su aparición estable en todos los grupos de edad.

La frecuencia de casos diagnosticados por cada 1000 citologías tomadas no mostró variación importante en relación con el grupo de edad excepto en las mayores de 45 años donde disminuyen. Al parecer las probabilidades de regresión o persistencia de las lesiones por PVH, NIC I y NIC II son semejantes hasta los 45 años, lo cual hablaría de un comportamiento similar de los tres tipos de lesiones y absolutamente diferente del NIC III y cáncer (Gráfico 5).

Otros autores (13) han comentado sobre el comportamiento similar de las NIC I y NIC II. Carson y Richard (14) notaron las discrepancias entre el comportamiento observado por las lesiones PVH, NIC I y NIC II con tendencias a presentarse con mayor frecuencia en mujeres jóvenes y el que revelaban los análisis utilizando la media como estadígrafo de tendencia central. En su estudio vieron que la distribución de frecuencias por edad y la moda describían con mejor aproximación a la realidad, las edades a las cuales estas lesiones se presentan con mayor frecuencia. La moda como estadígrafo no es influenciado por los valores extremos y reproduce con mayor fidelidad la pirámide etarea de la población que se investiga.

La aplicación de esta metodología de análisis permitió observar como 1 a distribución de frecuencias y las modas se desplazaban, en el caso de PVH y NIC I, de forma significativa hacia la izquierda, mientras que la NIC II y la NIC III coincidían con la distribución etarea de la población normal y la sometida a colposcopia. Esto permitió observar que las lesiones por PVH y NIC I son más frecuentes en edades jóvenes entre los 23 y 25 años.
El tamizaje en el grupo de mujeres entre 15 y 24 años traerá como consecuencia el diagnóstico de un alto volumen de lesiones de "bajo grado" poco predispuestas al cáncer (como lo revelan las cifras de incidencia y mortalidad a esta edad) que demandarán una atención y seguimiento no justificado pues la mayoría regresarán espontáneamente sin tratamiento como lo revelan los estudios sobre su evolución natural.

Se encuentra así una razón más que soporta la validez de la idea de evitar el tamizaje intensivo en menores de 25 años a riesgo de sobretratar y consumir los escasos recursos del programa en dos circunstancias, que a esa edad, no implican mayor riesgo.

De los análisis mostrados parecen desprenderse las siguientes ideas:

- Es prioritario proponerse alcanzar una cobertura del $100 \%$ en el grupo de 35 a 44 años de edad. La acción intensiva en este grupo permitirá obtener en el mediano plazo un descenso significativo de la incidencia y mortalidad de cáncer invasor.

- Es exagerado y poco eficaz el excesivo tamizaje en mujeres muy jóvenes; utiliza la cuarta parte del recurso en examinar este grupo de población por un riesgo mal entendido.

Se justifica plenamente la norma nacional de priorizar solo la población mayor de 25 años.

- Es dramática la disminución de la cobertura en la población mayor de 35 años; se requiere de un programa educativo y una orientación que desvincule la necesidad de la citología de los eventos reproductivos y que enfatice en mantener el tamizaje citológico a medida que la mujer envejece.

- No parece observarse cambios en el tiempo de evolución entre las lesiones preinvasoras y el cáncer.

- Las lesiones por P.V.H. y NIC I son más frecuentes en la población joven y la frecuencia relativa de P.V.H., NIC I y NIC II es constante por lo menos hasta los 45 años.

\section{Conclusiones}

1. Se identifican a las mujeres entre $\operatorname{los} 35$ y 44 años de edad como grupo prioritario para el programa de detección; en el mismo es mayor la frecuencia de NIC III y el carcinoma presenta un aumento significativo en relación al grupo de edad anterior. Del mismo modo fue el grupo que presentó mayor frecuencia de citología anormales.

2. La frecuencia de cáncer cérvico-uterino es bajo en edades tempranas por debajo de los 25 años, y antes de los 35 años todos los que se diagnosticaron fueron en estado I cuya sobrevida de cinco años oscila entre el 75 y $90 \%$.

3. Cerca de la cuarta parte de las citologías realizadas por Metrosalud durante los años 91-94 fueron en pacientes jóvenes menores de 25 años.

4. Las lesiones PVH y NIC I se obtuvieron con m mayor frecuencia absoluta en la población joven menor de 25 años, aunque la frecuencia relativa es constante en cada grupo de edad entre los 15 y los 44 años con un descanso importante en mujeres mayores de 45 años, muy diferente a las observadas con respecto a NIC III y cáncer de cuello uterino. 


\section{BIBLIOGRAFIA}

1. Instituto Nacional de Salud. División de Investigaciones especiales. Mortalidad en Colombia 1953-1994. 1994; 25.

2. Medina M. Atlas de Mortalidad por Cáncer. Colombia. 1990. Instituto Nacional de Cancerología, División de epidemiología. Santafé de Bogotá. 1994; 71.

3. Disaia P., Creasman W. Clinical Gynecologic Oncology. The C.V. Mosby Company, 1989; 73.

4. Instituto Nacional de Salud. Encuesta Nacional de Salud 1990; 155.

5. Idem (2) pág. 224.

6. Miller AB. Programas de detección del cáncer cérvico-uterino. Directrices de gestión. Organización Mundial de la Salud. Ginebra, 1993. NLM. WP 480. pág. 19.

7. Idem (6). pág. 16 .
8. Idem (6). pág. 17.

9. Idem (6). pág. 17.

10. Crowther ME. Is the Nature of Cervical Carcinoma changing in young Women? CME Review article. Obstetrical and Gynecological Survey. 1994; 50(1): 71-82.

11. Cramer DW., Cutler SS. Incidencia and histopathology of malignancies of the female genital organs in the United States. Am. J . Obstet. Gynecol. 1974; 118. 443.

12. Disaia P., Creasman W. Clinical Gynecologic oncology. The C.V. Mosby Company 1994

13. Idem (12). pág. 11.

14. Carson HH. and Richard MD. The mode ages of women with cervical dysplasia. Obstet and Gynecol. 1993; 82(3): 430. 